\title{
Intrarenal Distribution of Blood Flow in the Transplanted Dog Kidney: Effect of Denervation and Rejection *
}

\author{
Stanley M. Rosen, $†$ Bruno P. Truniger, $\ddagger$ Hans R. Kriek, Joseph E. Murray, \\ AND JoH N P. MERRILl \\ (From the Departments of Medicine and Surgery, Harvard Medical School and Peter Bent \\ Brigham Hospital, Boston, Mass.)
}

Summary. Serial measurements of intrarenal distribution of blood flow have been recorded in anesthetized dogs with the ${ }^{133}$ xenon "washout" technique. The results showed that normal kidneys redistributed their blood flow after laparotomy and mobilization of the kidney. This alteration consisted of a diminution in percentage of total renal blood flow supplied to the fastest flowing component, and a diminution of renal mass supplied by that component. This effect lasted for as long as 7 days. Thereafter, the blood flow distribution remained stable.

Autotransplanted kidneys had a stable distribution of blood flow between 0 and 77 days after operation, the values being identical with the stable normal kidney. Homotransplanted kidneys had the same intrarenal distribution of blood flow after operation as the autotransplanted kidneys. Whereas the intrarenal distribution of blood flow of the autotransplanted kidneys remained stable, a redistribution occurred in the homotransplanted kidneys as rejection progressed. . This phenomenon occurred before marked elevation of blood urea nitrogen.

The redistribution was due to a decrease in percentage of blood flow supplied to the fastest flowing component, and a relative reduction of tissue mass perfused by this component.

Radioautography of the kidneys before rejection demonstrated that the cortex was homogeneously perfused by the fastest flowing component of blood flow. As rejection progressed, a reduced area of cortex was perfused by this component. Terminally, the fastest flowing component was located in the outer medulla.

It is suggested that the reduction in cortical blood flow produced by immunological mechanisms may play a prominent role in the ensuing renal failure.

\section{Introduction}

Anatomical changes in the intrarenal vasculature are a prominent feature in the homotransplated

* Submitted for publication July 5, 1966; accepted April 13, 1967.

A partial report of this work was read at the Surgical Forum of the American College of Surgeons on October 20, 1965.

† Work performed during a medical fellowship from the Nuffield Foundation.

Address requests for reprints to Dr. Stanley M. Rosen, The General Infirmary, Leeds 1, England. kidney during rejection $(1,2)$. They consist of the accumulation of immunologically competent cells around small blood vessels and within the peritubular capillaries (1-3). These changes are most prominent in the cortex and, indeed, may be minimal in the medulla even at a late stage of rejection (1).

Such histological features may be associated with alterations in total renal blood flow. Kountz and

$¥$ Work performed during a fellowship from the Massachusetts Heart Association. 
co-workers (3) demonstrated a decrease in renal venous blood flow as rejection progressed. In the same group of experiments, they used the disappearance rate of intravenously injected hippuran${ }^{131} \mathrm{I}$ as a measurement of "effective renal plasma flow" and showed that this parameter was concomitantly decreased. Jackson and Mannick (4) measured renal blood flow with a sine-gated electromagnetic flowmeter placed around the renal artery of the dog and confirmed these observations.

No attempt has been made to determine whether this decrease in blood flow occurs uniformly throughout the kidney. In these experiments serial measurements of intrarenal distribution of blood flow were made with intra-arterial injections of ${ }^{133}$ xenon in dogs with normal kidneys, dogs with autotransplanted kidneys, and dogs with homotransplanted kidneys.

\section{Methods}

Theoretical considerations in determination of intrarenal distribution of blood flow. A bolus of ${ }^{133}$ xenon injected into the major renal artery is distributed among the intrarenal components of blood flow. The quantity of xenon received by each of these components is directly proportional to the fraction of total renal blood flow con-

COMPARTMENTAL DISTRIBUTION OF BLOOD FLOW IN THE NORMAL DOG KIDNEY

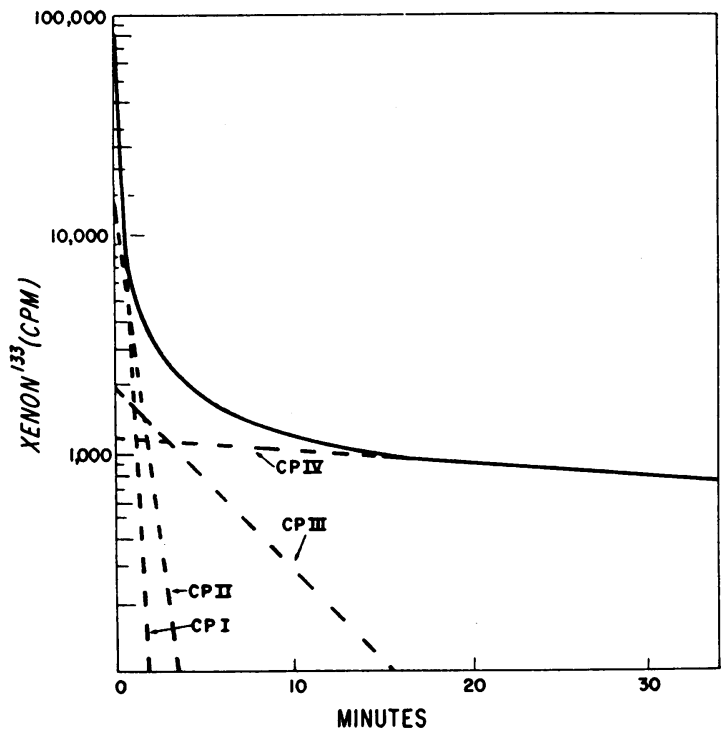

Fig. 1. CURVE obtained BY EXTERNAL MONItoring OVER NORMAL DOG KIDNEY AFTER INJECTION OF BOLUS OF ${ }^{133}$ XENON INTO A RENAL ARTERY. The curve was analyzed graphically as the sum of four exponential components. These are represented by interrupted lines. stituting the respective component. The xenon attains almost instantaneous equilibrium between blood and parenchyma (5) and can only be removed from the parenchyma by urine, lymph, and capillary blood flow. Since urine and lymph flow are small compared with blood flow, the rate of disappearance of the xenon from the renal parenchyma is a measure of capillary blood flow.

If it is assumed that the major blood flow components are arranged in parallel within the kidney, then the quantity of ${ }^{183}$ xenon (A) remaining in the renal parenchyma at time $t$ after injection of the bolus is represented by $\mathrm{A}=\mathrm{A}_{0} \mathrm{I}_{0} \mathrm{e}^{-\mathrm{k} \mathrm{I}_{\mathrm{t}}}+\mathrm{A}^{\mathrm{II}} \mathrm{e}^{-\mathrm{kII} t} \cdots+\mathrm{A}_{0} \mathrm{e}^{-\mathrm{k}^{\pi} \mathrm{t}}(6,7)$, where $\mathrm{A}_{0}{ }^{\pi}$ represents the amount of radioactivity supplied to component $\pi$ and $-\mathrm{k}^{\pi}$ is the rate constant of removal of radioactivity by that component. Values for $A_{0}{ }^{\pi}$ and $k^{\pi}$ can be determined by graphical analysis of a semilogarithmic plot of total radioactivity against time (8). In the normal kidney four exponential components can be separated (Figure 1). A straight line is drawn by inspection through the terminal portion of the curve and extrapolated to zero time. This line is component IV, its intecept reperesents $\mathrm{A}_{0}{ }^{\mathrm{IV}}$, and the slop of the line is $-\mathrm{k}^{\mathbf{I V}}$. The quantity of xenon represented at each point in time on the extrapolated line is subtracted from the original curve, and a new curve is constructed whose terminal portion represents component III. Components II and I are derived in sequential fashion. Thorburn and co-workers (6) have demonstrated that computer analysis of the curve gives excellent agreement with this graphical analysis. The fraction of total renal blood flow supplied to each component is calculated from the amount of xenon in each component at zero time divided by the quantity of xenon in all four components at zero time. Flow per unit mass of tissue in each component is derived from the respective decay constant ( $k$ ) by using the Kety application of the Fick principle (9). Thus, $F=(k \times \lambda \times 100) / \xi$, where $\mathrm{F}$ is the flow rate in milliliters per $100 \mathrm{~g}$ tissue per minute, $\mathrm{k}$ the slope of the line, $\lambda$ the partition coefficient for ${ }^{138}$ xenon between tissue and blood, and $\xi$ the specific gravity of the tissue. Lambda is 0.65 for renal tissue and 7.65 for fat $(10)$.

The relative mass of tissue supplied by each component is obtained by dividing the quantity of xenon supplied by each component $\left(A_{0}\right)$ by the rate constant of removal of xenon for that component (k) (7). Thus, the ratio of mass of tissue perfused by component $I\left(\mathrm{M}^{\mathrm{I}}\right)$ divided by the mass of tissue perfused by component II $\left(\mathrm{M}^{\mathrm{II}}\right)=$ $\left[\left(\mathrm{A}^{\mathrm{I}} \mathrm{o} / \mathrm{k}^{\mathrm{I}} /\left(\mathrm{A}^{\mathrm{II}}{ }_{0} / \mathrm{k}^{\mathrm{II}}\right)\right]\right.$.

Since $\mathrm{A}^{\mathrm{III}}$ and $\mathrm{A}^{\mathrm{IV}}{ }_{0}$ are small compared to $\mathrm{A}^{\mathrm{I}}{ }_{0}$ and $\mathrm{A}^{\mathrm{II}}{ }_{0}$, the percentage of renal tissue perfused by component $\mathrm{I}=$ $\left[\left(\mathrm{A}^{\mathrm{I}}{ }_{0} / \mathrm{k}^{\mathrm{I}} /\left(\mathrm{A}^{\mathrm{I}}{ }_{0} / \mathrm{k}^{\mathrm{I}}+\mathrm{A}^{\mathrm{II}}{ }_{0} / \mathrm{k}^{\mathrm{II}}\right)\right](12)\right.$.

Validation of the inert gas washout technique for the measurement of regional blood flow in kidney has been confirmed by several groups (11-13); components I, II, III, and IV in the normal kidney are localized to the cortex, outer medulla, inner medulla, and renal fat, respectively (6). However, certain limitations of interpretation of the curve must be considered during abnormal situations. Anatomical localization of blood flow com- 
ponents may be inconstant and would have to be validated on each occasion. Unstable areas of blood flow may produce problems of curve analysis, since under these conditions the curve does not consist of a series of graded exponentials. Arteriovenous "shunts" are not detected with this technique, because only blood flowing through capillaries is capable of "washing" out xenon from the parenchyma.

Procedure. Three groups of mongrel dogs weighing between 14 and $21 \mathrm{~kg}$ were used for the experiments.

Group 1 consisted of 16 dogs with normal kidneys. The left renal artery was exposed through a midline incision. A catheter of polyvinyl chloride tubing was introduced into the artery by the technique of Herd and Barger (14). The $1-\mathrm{cm}$ length of catheter remaining within the arterial lumen had previously been stretched so that its external diameter was 0.5 to $0.8 \mathrm{~mm}$ and it did not obstruct blood flow. The catheter was secured by a stitch that juxtaposed the arterial adventitia to a collar surrounding the catheter. The other end of the catheter was passed through the abdominal wall. Patency of the catheter lumen was maintained by refilling the catheter daily with heparin (1,000 $\mathrm{U}$ per $\mathrm{ml})$.

Group 2 consisted of 15 dogs with a kidney autotransplanted in the right iliac fossa. An end-to-end anastomosis of the renal and common iliac arteries and an end-toside anastomosis of renal and iliac veins were performed, and the ureter was reimplanted into the bladder. A catheter was sutured in the common iliac artery supplying the transplant and threaded through a stab incision in the abdominal wall. The ischemic time was less than $45 \mathrm{~min}$ utes, and no attempt was made to perfuse the kidney during this period.

Group 3 consisted of 12 dogs. Each dog had a homotransplanted kidney inserted in the right iliac fossa and a catheter placed in the right common iliac artery, using the same technique as described for Group 2. At the same operation the dogs' own kidneys were removed. Blood urea nitrogen was serially estimated before and after this procedure with the Technicon autoanalyzer.

Serial measurements of the intrarenal distribution of blood flow (IDBF) were made in the three groups of dogs at a constant level of pentobaribital anesthesia. The initial dose was given slowly in a foreleg vein until the outer canthal reflex disappeared. Additional pentobarbital was given intermittently to maintain a constant depth of anesthesia.

A bolus of 200 to $400 \mu \mathrm{c}$ of ${ }^{138}$ xenon dissolved in less than $0.5 \mathrm{mi}$ isotonic saline was injected into the arterial catheter.

The rate of disappearance of the ${ }^{138}$ xenon was externally monitored by a scintillation probe with a 2 -inch sodium iodide crystal placed 3 inches from the end of a cylindrical collimator. The output from the probe was led into either a linear ratemeter connected to a linear chart recorder or a digital ratemeter connected to a digital printer.

The time constant for the linear ratemeter was $0.5 \mathrm{sec}-$ ond for the first minute, 2.5 seconds for the second and third minutes, 10 seconds for the fourth and fifth minutes, and 40 seconds for the remainder of the 45 -minute curve. The chart recorder speed was 12 inches per minute for the first 5 minutes and 0.75 for the subsequent 40 minutes.

The digital ratemeter and printer, respectively, integrated and recorded counts every 2 seconds for the first minute, every 6 seconds for the second and third minutes, every 20 seconds for the fourth and fifth minutes, and every minute for the subsequent 40 minutes.

Counts per minute were plotted on a logarithmic scale every 2 seconds for the first minute, every $6 \mathrm{sec}-$ onsd for the second and third minutes, every 20 seconds for the fourth and fifth minutes, and at minute intervals thereafter. Four exponential components representing four blood flow components were derived from this curve as previously described (8) (Figure 1).

- Some measurements were made with simultaneous recordings of the probe output by the linear and digital recording systems. The resultant curves produced identical results.

Since the counts per minute recorded by the external counting system were proportional to the quantity of xenon, the fraction of total renal blood flow that constituted each component was calculated by dividing the number of counts in that component at zero time by the sum of the counts in all four components at zero time. The blood flow per unit mass of tissue in each component was calculated from the rate of decay of activity in the component.

Radioautographs of four normal, three autotransplanted, and seven homotransplated kidneys were performed at selected intervals after operation. The animal was anesthetized to the prescribed standard, and the intrarenal distribution of blood flow was measured with ${ }^{188}$ xenon. the renal vessels were then exposed without disturbing the kidney. A bolus of 200 to $400 \mu \mathrm{c}{ }^{80} \mathrm{krypton}$ dissolved in $0.5 \mathrm{ml}$ saline was injected through the renal arterial catheter, and a ligature was immediately tied around the renal vessels. The kidney was rapidly removed and frozen to $-40^{\circ} \mathrm{C}$ in a mixture of dry ice and acetone and maintained at this temperautre for the rest of the procedure. Coronal sections of kidney $1 \mathrm{~mm}$ thick were then exposed on Kodak AA industrial x-ray paper, with exposure times of $\frac{1}{2}$ to 6 hours. The kidney slices were then photographed so that anatomical boundaries could be superimposed on the radioautograph. Results were obtained from a minimum of five slices from each kidney.

${ }^{85}$ Krypton was used for radioautography, since it is predominantly a beta ray emitter. However, xenon and krypton share the same properties described as prerequisites for application of the method $(15,16)$. Thus, the components represented in the ${ }^{183}$ xenon washout curves were the same as those represented in the ${ }^{80}$ krypton radioautographs.

\section{Results}

Normal kidneys. Sixteen measurements made in 13 dogs less than 7 days after mobilization of the kidney and insertion of renal arterial catheter are shown in Table I. The mean percentages of 
TABLE I

Normal dog kidney within 7 days of operation to mobilize kidney and insert renal arterial catheter*

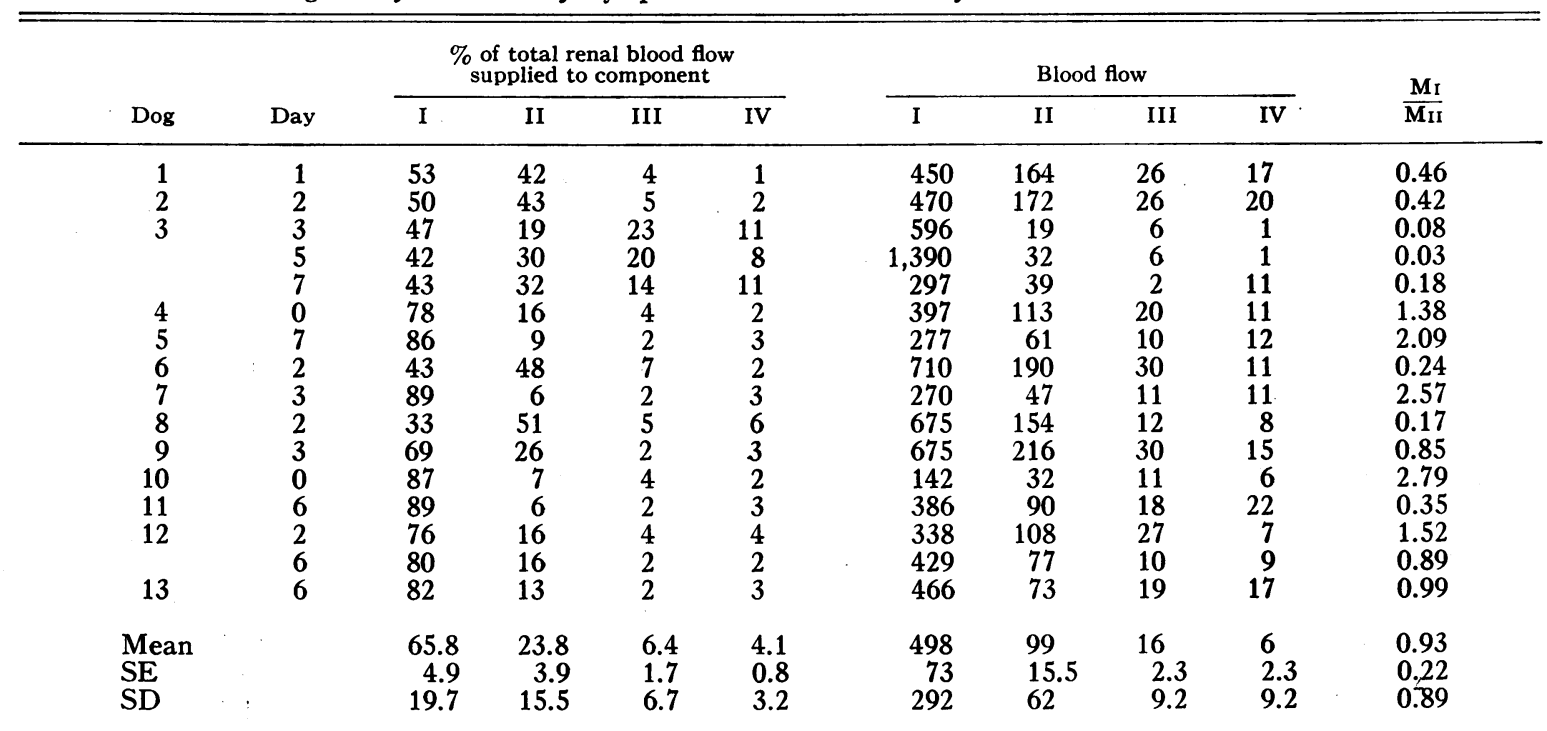

* Measurements of percentage of total renal blood flow supplied to each component $\left(A_{0}\right)$ and blood flow in milliliters per $100 \mathrm{~g}$ tissue per minute for each component $(\mathrm{k})$ are shown. Ratio of mass of tissue supplied by component I divided by mass of tissue supplied by component II has been calculated $\left(\mathrm{M}_{\mathrm{I}} / \mathrm{M}_{\mathrm{II}}\right)$.

total renal blood flow supplied to components I, II, the size of the tissue mass supplied by component III, and IV, respectively, were $65.8 \pm 4.9 \% \mathrm{SE}$, $23.8 \pm 3.9 \%, 6.4 \pm 1.7 \%$, and $4.1 \pm 0.8 \%$. The mean blood flow per $100 \mathrm{~g}$ tissue per minute was $498,99,16$, and $6 \mathrm{ml}$, respectively. The tissue mass supplied by component I was $0.93 \pm 0.22 \mathrm{SE}$. flow supplied to components I, II, III, and IV, re-

Eighteen measurements in eight dogs made more than 7 days after this operation are shown in Table II. The mean percentages of total renal blood TABLE II

Normal dog kidney more than 7 days after operation to mobilize kidney and insert renal arterial catheter

\begin{tabular}{|c|c|c|c|c|c|c|c|c|c|c|}
\hline \multirow[b]{2}{*}{ Dog } & \multirow[b]{2}{*}{ Day } & \multicolumn{4}{|c|}{$\begin{array}{l}\text { \% of total renal blood flow } \\
\text { supplied to component }\end{array}$} & \multicolumn{4}{|c|}{ Blood flow } & \multirow{2}{*}{$\frac{\mathrm{M}_{\mathrm{I}}}{\mathbf{M}_{\mathrm{II}}}$} \\
\hline & & I & II & III & IV & I & II & III & IV & \\
\hline 3 & 16 & 85 & 12 & 2 & 1 & 338 & 82 & 5 & 9 & 1.72 \\
\hline \multirow[t]{4}{*}{5} & 11 & 79 & 12 & 5 & 4 & 338 & 95 & 16 & 12 & 1.85 \\
\hline & 37 & 81 & 13 & 2 & 4 & 310 & 110 & 18 & 9 & 2.21 \\
\hline & 88 & 90 & 3 & 3 & 4 & 193 & 45 & 8 & 5 & 7.06 \\
\hline & 120 & 71 & 19 & 3 & 3 & 386 & 113 & 15 & 5 & 1.57 \\
\hline \multirow[t]{2}{*}{9} & 24 & 86 & 7 & 4 & 3 & 482 & 93 & 17 & 7 & 2.37 \\
\hline & 64 & 80 & 13 & 4 & 3 & 190 & 53 & 10 & 8 & 1.72 \\
\hline \multirow{3}{*}{$\begin{array}{l}10 \\
11\end{array}$} & 31 & 90 & 5 & 3 & 2 & 200 & 41 & 13 & 12 & 3.68 \\
\hline & 20 & 82 & 13 & 2 & 3 & 562 & 128 & 8 & 8 & 1.43 \\
\hline & 20 & 72 & 24 & 2 & 2 & 450 & 135 & 11 & 7 & 0.90 \\
\hline \multirow[t]{4}{*}{14} & 26 & 89 & 8 & 2 & 1 & 540 & 104 & 16 & 7 & 2.14 \\
\hline & 28 & 89 & 8 & 2 & 1 & 600 & 142 & 14 & 19 & 2.64 \\
\hline & 33 & 79 & 17 & 2 & 2 & 900 & 142 & 16 & 10. & 0.73 \\
\hline & 54 & 85 & 13 & 1 & 1 & 818 & 90 & 12 & 7 & 0.72 \\
\hline \multirow{4}{*}{$\begin{array}{l}15 \\
16\end{array}$} & 12 & 89 & 6 & 3 & 2 & 338 & 69 & 18 & 13 & 3.05 \\
\hline & 36 & 85 & 9 & 2 & 4 & 163 & 50 & 5 & 8 & 2.89 \\
\hline & 50 & 83 & 12 & 3 & 2 & 164 & 60 & 11 & 4 & 2.53 \\
\hline & 84 & 85 & 7 & 3 & 5 & 251 & 71 & 16 & 8 & 3.76 \\
\hline Mean & & 83.6 & 11.1 & 2.6 & 2.6 & 395 & 91 & 13 & 9 & 2.39 \\
\hline SE & & 1.2 & 1.2 & 0.7 & 0.3 & 48 & 8.4 & 0.95 & 0.8 & 0.35 \\
\hline SD & & 5.3 & 5.1 & 0.95 & 1.21 & 209 & 37 & 4.2 & 3.5 & 1.47 \\
\hline
\end{tabular}


TABLE III

Dog kidney within 7 days of autotransplantation

\begin{tabular}{|c|c|c|c|c|c|c|c|c|c|c|}
\hline \multirow[b]{2}{*}{ Dog } & \multirow[b]{2}{*}{ Day } & \multicolumn{4}{|c|}{$\begin{array}{l}\% \text { of total renal blood flow } \\
\text { supplied to component }\end{array}$} & \multicolumn{4}{|c|}{ Blood flow } & \multirow{2}{*}{$\frac{\mathbf{M}_{\mathbf{I}}}{\mathrm{M}_{\mathrm{II}}}$} \\
\hline & & I & II & III & IV & I & II & III & IV & \\
\hline $\begin{array}{r}1 \\
4 \\
7 \\
9 \\
11 \\
17 \\
18 \\
19 \\
20 \\
21 \\
22\end{array}$ & $\begin{array}{l}1 \\
0 \\
3 \\
7 \\
6 \\
1 \\
2 \\
0 \\
2 \\
0 \\
3\end{array}$ & $\begin{array}{l}84 \\
86 \\
82 \\
92 \\
76 \\
89 \\
88 \\
77 \\
68 \\
84 \\
88\end{array}$ & $\begin{array}{r}10 \\
11 \\
10 \\
5 \\
20 \\
9 \\
8 \\
21 \\
26 \\
13 \\
10\end{array}$ & $\begin{array}{l}5 \\
2 \\
4 \\
2 \\
2 \\
1 \\
1 \\
1 \\
5 \\
2 \\
1\end{array}$ & $\begin{array}{l}1 \\
1 \\
4 \\
1 \\
2 \\
1 \\
3 \\
1 \\
1 \\
1 \\
1\end{array}$ & $\begin{array}{l}193 \\
416 \\
193 \\
600 \\
386 \\
587 \\
456 \\
300 \\
563 \\
491 \\
772\end{array}$ & $\begin{array}{r}63 \\
132 \\
51 \\
75 \\
90 \\
89 \\
59 \\
117 \\
90 \\
69 \\
146\end{array}$ & $\begin{array}{r}22 \\
16 \\
12 \\
25 \\
18 \\
16 \\
9 \\
9 \\
17 \\
20 \\
16\end{array}$ & $\begin{array}{r}17 \\
23 \\
17 \\
20 \\
22 \\
10 \\
5 \\
5 \\
19 \\
19 \\
15\end{array}$ & $\begin{array}{l}2.75 \\
2.48 \\
2.17 \\
2.32 \\
0.89 \\
1.50 \\
1.83 \\
1.44 \\
0.42 \\
0.91 \\
1.67\end{array}$ \\
\hline $\begin{array}{l}\text { Mean } \\
\text { SE } \\
\text { SD }\end{array}$ & & $\begin{array}{r}80.7 \\
2.0 \\
7.7\end{array}$ & $\begin{array}{r}15.1 \\
1.8 \\
7.1\end{array}$ & $\begin{array}{l}3.1 \\
0.6 \\
2.3\end{array}$ & $\begin{array}{l}1.5 \\
0.3 \\
1.0\end{array}$ & $\begin{array}{r}492 \\
54 \\
210\end{array}$ & $\begin{array}{r}102 \\
10 \\
37\end{array}$ & $\begin{array}{r}18 \\
2 \\
6\end{array}$ & $\begin{array}{r}15 \\
2 \\
6\end{array}$ & $\begin{array}{l}1.67 \\
0.22 \\
0.73\end{array}$ \\
\hline
\end{tabular}

spectively, were $83.6 \pm 1.2 \% \mathrm{SE}, 11.1 \pm 1.2 \%$, $2.6 \pm 0.7 \%$, and $2.6 \pm 0.3 \%$. The mean blood flow per $100 \mathrm{~g}$ tissue per minute for each of these components was $395,91,13$, and $9 \mathrm{ml}$, respectively. The tissue mass supplied by component I was $2.39 \pm 0.35 \mathrm{SE}$ greater than that supplied by component II. The difference between the means of the intrarenal distribution of blood flow when measured before and after 7 days are significant at $p<0.001, p<0.01$, and $\mathrm{p}<0.02$ for components I, II, and III, respec- tively. However, the mean blood flow rates per $100 \mathrm{~g}$ tissue for these compartments were not significantly different when measured before or after the seventh postoperative day. Relatively more tissue was perfused by component I after the seventh postoperative day $(\mathrm{p}<0.01)$.

Autotransplanted kidneys. Eleven measurements in 11 dogs made less than 7 days after operation are shown in Table III. The mean percentages of total renal blood flow supplied to components I, II, III, and IV were, respectively,

TABLE IV

Dog kidney more than 7 days after autotransplantation

\begin{tabular}{|c|c|c|c|c|c|c|c|c|c|c|}
\hline \multirow[b]{2}{*}{ Dog } & \multirow[b]{2}{*}{ Day } & \multicolumn{4}{|c|}{$\begin{array}{l}\% \text { of total renal blood flow } \\
\text { supplied to component }\end{array}$} & \multicolumn{4}{|c|}{ Blood flow } & \multirow{2}{*}{$\frac{\mathbf{M}_{\mathrm{I}}}{\mathbf{M}_{\mathrm{II}}}$} \\
\hline & & I & II & III & IV & I & II & III & IV & \\
\hline $\begin{array}{r}5 \\
\\
7 \\
9 \\
11 \\
16 \\
17 \\
18 \\
20\end{array}$ & $\begin{array}{r}11 \\
13 \\
37 \\
37 \\
10 \\
20 \\
36 \\
8 \\
11 \\
17 \\
10 \\
14 \\
30 \\
30 \\
77 \\
8 \\
10 \\
23 \\
40\end{array}$ & $\begin{array}{l}76 \\
72 \\
90 \\
81 \\
92 \\
79 \\
85 \\
79 \\
76 \\
75 \\
78 \\
76 \\
69 \\
67 \\
87 \\
77 \\
73 \\
81 \\
87\end{array}$ & $\begin{array}{r}19 \\
19 \\
6 \\
13 \\
4 \\
18 \\
9 \\
15 \\
21 \\
18 \\
20 \\
20 \\
26 \\
29 \\
9 \\
12 \\
11 \\
16 \\
10\end{array}$ & $\begin{array}{l}2 \\
4 \\
1 \\
5 \\
2 \\
2 \\
2 \\
2 \\
2 \\
3 \\
1 \\
3 \\
4 \\
2 \\
3 \\
6 \\
8 \\
1 \\
2\end{array}$ & $\begin{array}{l}3 \\
5 \\
3 \\
1 \\
2 \\
1 \\
4 \\
4 \\
1 \\
4 \\
1 \\
1 \\
1 \\
2 \\
1 \\
5 \\
8 \\
2 \\
2\end{array}$ & $\begin{array}{r}370 \\
169 \\
257 \\
252 \\
600 \\
450 \\
163 \\
474 \\
540 \\
386 \\
675 \\
750 \\
676 \\
568 \\
563 \\
900 \\
1,080 \\
901 \\
300\end{array}$ & $\begin{array}{r}110 \\
78 \\
84 \\
91 \\
75 \\
100 \\
50 \\
150 \\
142 \\
77 \\
159 \\
154 \\
234 \\
204 \\
108 \\
62 \\
37 \\
260 \\
77\end{array}$ & $\begin{array}{r}14 \\
11 \\
19 \\
20 \\
25 \\
14 \\
5 \\
35 \\
18 \\
20 \\
10 \\
10 \\
36 \\
20 \\
30 \\
10 \\
14 \\
9 \\
17\end{array}$ & $\begin{array}{r}14 \\
25 \\
6 \\
28 \\
20 \\
9 \\
4 \\
10 \\
15 \\
12 \\
12 \\
17 \\
26 \\
16 \\
18 \\
11 \\
11 \\
6 \\
9\end{array}$ & $\begin{array}{l}1.19 \\
1.75 \\
4.92 \\
2.26 \\
2.88 \\
0.98 \\
2.89 \\
1.67 \\
0.95 \\
0.83 \\
0.93 \\
0.78 \\
0.92 \\
0.83 \\
1.85 \\
0.44 \\
0.23 \\
1.44 \\
2.24\end{array}$ \\
\hline $\begin{array}{l}\text { Mean } \\
\text { SE } \\
\text { SD }\end{array}$ & & $\begin{array}{l}79 \\
1.6 \\
6.8\end{array}$ & $\begin{array}{r}15.5 \\
1.5 \\
6.6\end{array}$ & $\begin{array}{l}2.9 \\
0.4 \\
1.9\end{array}$ & $\begin{array}{l}2.6 \\
0.4 \\
1.9\end{array}$ & $\begin{array}{r}530 \\
60 \\
262\end{array}$ & $\begin{array}{r}119 \\
14 \\
62\end{array}$ & $\begin{array}{r}18 \\
1.9 \\
8.4\end{array}$ & $\begin{array}{r}14 \\
1.6 \\
6.9\end{array}$ & $\begin{array}{l}1.57 \\
0.26 \\
1.11\end{array}$ \\
\hline
\end{tabular}


TABLE V

Dog kidney less than 2 hours after first set homotransplantation

\begin{tabular}{|c|c|c|c|c|c|c|c|c|c|c|}
\hline \multirow[b]{2}{*}{ Dog } & \multicolumn{4}{|c|}{$\begin{array}{c}\% \text { of total renal blood flow } \\
\text { supplied to component }\end{array}$} & \multicolumn{4}{|c|}{ Blood flow } & \multirow{2}{*}{$\frac{\mathbf{M}_{\mathrm{I}}}{\overline{\mathbf{M}_{\mathrm{II}}}}$} & \multirow{2}{*}{$\frac{M_{I}}{M_{I}+M_{I I}} \times 100 \% *$} \\
\hline & I & II & III & IV & I & II & III & IV & & \\
\hline $\begin{array}{l}25 \\
26 \\
27 \\
28 \\
29 \\
30 \\
31 \\
32 \\
33 \\
34 \\
35 \\
36\end{array}$ & $\begin{array}{l}89 \\
80 \\
89 \\
78 \\
73 \\
79 \\
75 \\
78 \\
88 \\
73 \\
85 \\
83\end{array}$ & $\begin{array}{r}9 \\
16 \\
8 \\
20 \\
23 \\
19 \\
22 \\
16 \\
10 \\
23 \\
13 \\
14\end{array}$ & $\begin{array}{l}1 \\
2 \\
1 \\
1 \\
2 \\
1 \\
2 \\
4 \\
1 \\
3 \\
1 \\
2\end{array}$ & $\begin{array}{l}1 \\
2 \\
2 \\
1 \\
2 \\
1 \\
1 \\
2 \\
1 \\
1 \\
1 \\
1\end{array}$ & $\begin{array}{l}540 \\
270 \\
237 \\
314 \\
360 \\
234 \\
257 \\
371 \\
322 \\
429 \\
422 \\
659\end{array}$ & $\begin{array}{r}86 \\
61 \\
57 \\
86 \\
75 \\
71 \\
104 \\
111 \\
90 \\
150 \\
87 \\
200\end{array}$ & $\begin{array}{r}12 \\
14 \\
12 \\
8 \\
11 \\
11 \\
13 \\
18 \\
14 \\
24 \\
25 \\
35\end{array}$ & $\begin{array}{r}9 \\
17 \\
14 \\
14 \\
5 \\
13 \\
9 \\
11 \\
12 \\
14 \\
12 \\
25\end{array}$ & $\begin{array}{l}1.58 \\
1.12 \\
2.68 \\
1.01 \\
0.66 \\
1.26 \\
1.37 \\
1.45 \\
2.39 \\
1.11 \\
1.25 \\
1.78\end{array}$ & $\begin{array}{l}\mathbf{6 1 . 2} \\
52.8 \\
72.8 \\
50.3 \\
39.7 \\
55.8 \\
57.8 \\
59.2 \\
70.5 \\
52.6 \\
55.6 \\
64.0\end{array}$ \\
\hline $\begin{array}{l}\text { Mean } \\
\text { SE } \\
\text { SD }\end{array}$ & $\begin{array}{r}79.0 \\
1.7 \\
5.8\end{array}$ & $\begin{array}{r}15.5 \\
1.5 \\
5.3\end{array}$ & $\begin{array}{l}2.9 \\
0.16 \\
0.57\end{array}$ & $\begin{array}{l}2.6 \\
0.16 \\
0.57\end{array}$ & $\begin{array}{l}530 \\
118 \\
408\end{array}$ & $\begin{array}{r}119 \\
13 \\
45\end{array}$ & $\begin{array}{c}18 \\
2.2 \\
7.8\end{array}$ & $\begin{array}{c}14 \\
1.4 \\
4.9\end{array}$ & $\begin{array}{l}1.47 \\
0.17 \\
0.57\end{array}$ & $\begin{array}{r}57.7 \\
2.6 \\
8.9\end{array}$ \\
\hline
\end{tabular}

* Renal mass perfused by component I.

$80.7 \pm 2.0 \%$ SE, $15.1 \pm 1.8 \%, 3.1 \pm 0.6 \%$, and $1.6 \pm 0.3 \%$. The mean blood flows per $100 \mathrm{~g}$ tissue per minute in these components were 492, 102,18 , and $15 \mathrm{ml}$, respectively. The tissue mass supplied by component I was $1.67 \pm 0.22 \mathrm{SE}$ greater than that supplied by component II.

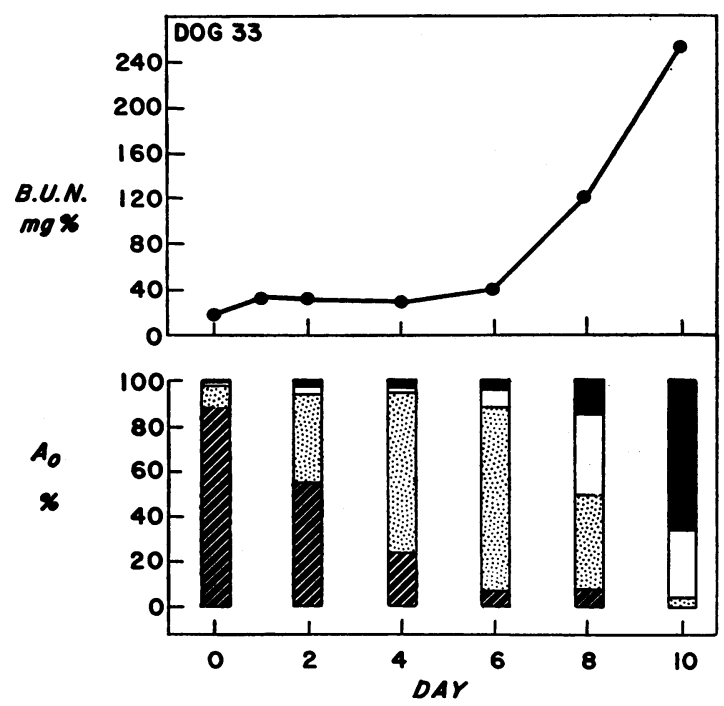

Fig. 2. Serial measurements of Intrarenal distriBUTION OF BLOOD FLOW IN THE HOMOTRANSPLANTED KIDNEY OF A BILATERALLY NePhReCTOMIZED DOG. Percentages of total renal blood flow $\left(\mathrm{A}_{0}\right)$ received by components $\mathrm{I}$, II, III, and IV are represented by crosshatched, dotted, white, and black blocks, respectively. Alteration in component I occurred before elevation of blood urea nitrogen (BUN)
These results were not significantly different from the means of 19 measurements made in 10 dogs with autotransplanted kidneys more than 7 days after operation (Table IV), nor from the results obtained in normal kidneys more than 7 days postoperatively. However, the distribution of

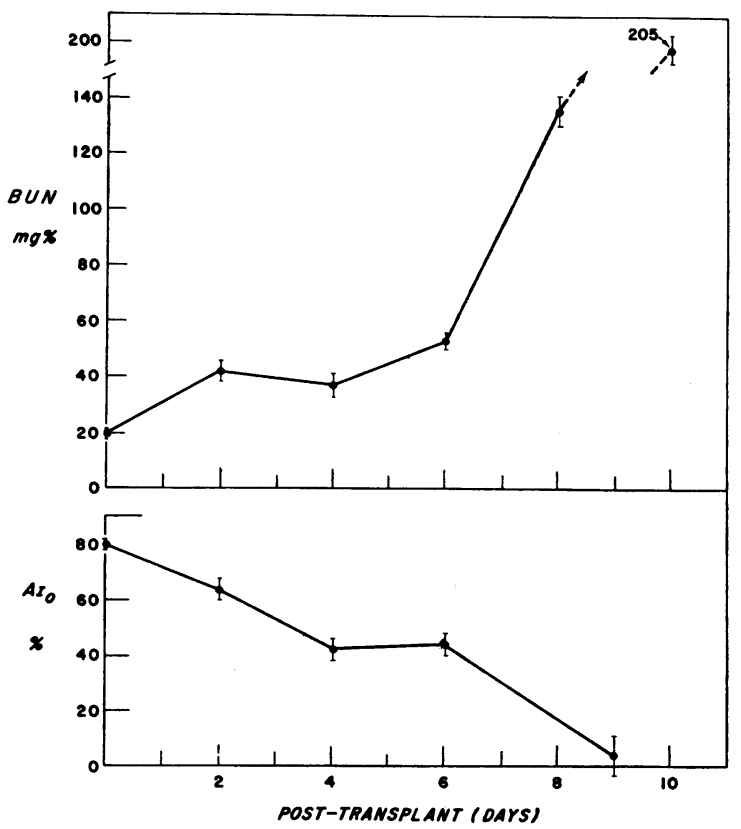

Fig. 3. RELATIONShip OF MEAN VALUES AND STANDARD ERRORS FOR BUN AND PERCENTAGE OF TOTAL RENAL BLOOD FLOW RECEIVED BY COMPONENT I ( $\mathrm{AI}_{0}$ ) OF HOMOTRANSPLANTED KIDNEY IN A GROUP OF BILATERALLY NEPHRECTOMIZED DOGS. 
blood flow in the autotransplanted kidneys within pletion of the vascular anastomoses are shown in

7 days of operation differed significantly from that in the normal kidneys within the first 7 postoperative days $(\mathrm{p}<0.01)$.

Homotransplanted kidneys. The values of measurements made in 12 dogs within 2 hours of com-
Table V. The mean of these values did not differ significantly from the mean values in the autotransplanted group from 0 to 7 days postoperatively, but showed a significantly greater percentage of renal blood flow supplied to component I

TABLE VI

Changes in intrarenal distribution of blood flow and blood urea nitrogen ( $B U N$ ) during rejection of a homotransplanted kidney

\begin{tabular}{|c|c|c|c|c|c|c|c|c|c|c|c|c|c|}
\hline \multirow[b]{2}{*}{ Dog } & \multirow[b]{2}{*}{ Day } & \multicolumn{4}{|c|}{$\begin{array}{c}\% \text { of total renal blood } \\
\text { flow supplied to } \\
\text { component }\end{array}$} & \multicolumn{4}{|c|}{ Blood flow } & \multirow{2}{*}{$\frac{M_{1}}{M_{1 I}}$} & \multirow{2}{*}{$\frac{M_{I}}{\underset{\times 100 \%}{M_{I}+M_{I I}}}$} & \multirow[b]{2}{*}{ BUN } & \multirow{2}{*}{ Radioautog } \\
\hline & & I & II & III & IV & I & II & III & IV & & & & \\
\hline 26 & $\begin{array}{l}0 \\
3 \\
5 \\
8\end{array}$ & $\begin{array}{l}80 \\
76 \\
58 \\
18\end{array}$ & $\begin{array}{l}16 \\
20 \\
37 \\
33\end{array}$ & $\begin{array}{r}3 \\
2 \\
4 \\
25\end{array}$ & $\begin{array}{r}2 \\
2 \\
1 \\
24\end{array}$ & $\begin{array}{l}270 \\
246 \\
257 \\
346\end{array}$ & $\begin{array}{r}61 \\
87 \\
113 \\
39\end{array}$ & $\begin{array}{l}14 \\
17 \\
18 \\
11\end{array}$ & $\begin{array}{l}17 \\
15 \\
18 \\
10\end{array}$ & $\begin{array}{l}1.12 \\
1.33 \\
0.69 \\
0.06\end{array}$ & $\begin{array}{r}52.8 \\
56.9 \\
40.6 \\
5.9\end{array}$ & $\begin{array}{r}12 \\
59 \\
35 \\
216\end{array}$ & \\
\hline 27 & $\begin{array}{l}0 \\
2 \\
3 \\
6 \\
8 \\
9\end{array}$ & $\begin{array}{l}80 \\
73 \\
31 \\
20\end{array}$ & $\begin{array}{l}17 \\
22 \\
45 \\
54\end{array}$ & $\begin{array}{r}1 \\
2 \\
19 \\
22\end{array}$ & $\begin{array}{l}2 \\
3 \\
5 \\
4\end{array}$ & $\begin{array}{r}349 \\
221 \\
142 \\
98\end{array}$ & $\begin{array}{l}71 \\
82 \\
54 \\
55\end{array}$ & $\begin{array}{l}14 \\
15 \\
17 \\
16\end{array}$ & $\begin{array}{l}14 \\
19 \\
15 \\
14 \\
23\end{array}$ & $\begin{array}{l}2.68 \\
0.93 \\
1.26 \\
0.27 \\
0.20\end{array}$ & $\begin{array}{l}72.8 \\
48.5 \\
59.3 \\
20.8 \\
16.9\end{array}$ & $\begin{array}{r}34 \\
68 \\
64 \\
176 \\
237\end{array}$ & \\
\hline 28 & $\begin{array}{l}0 \\
2\end{array}$ & $\begin{array}{l}78 \\
65\end{array}$ & $\begin{array}{l}20 \\
28\end{array}$ & $\begin{array}{l}1 \\
5\end{array}$ & $\begin{array}{l}1 \\
2\end{array}$ & $\begin{array}{l}314 \\
142\end{array}$ & $\begin{array}{l}86 \\
59\end{array}$ & $\begin{array}{r}8 \\
15\end{array}$ & $\begin{array}{l}14 \\
25\end{array}$ & $\begin{array}{l}1.01 \\
0.97\end{array}$ & $\begin{array}{l}50.0 \\
49.2\end{array}$ & $\begin{array}{l}18 \\
54\end{array}$ & \\
\hline $29 *$ & $\begin{array}{r}0 \\
2 \\
4 \\
6 \\
8 \\
10\end{array}$ & $\begin{array}{l}73 \\
82 \\
40 \\
24 \\
17\end{array}$ & $\begin{array}{l}23 \\
13 \\
53 \\
69 \\
73 \\
80\end{array}$ & $\begin{array}{r}2 \\
3 \\
5 \\
3 \\
8 \\
15\end{array}$ & $\begin{array}{l}2 \\
2 \\
2 \\
4 \\
2 \\
5\end{array}$ & $\begin{array}{l}360 \\
338 \\
520 \\
529 \\
750\end{array}$ & $\begin{array}{r}75 \\
82 \\
139 \\
87 \\
102 \\
59\end{array}$ & $\begin{array}{l}11 \\
24 \\
28 \\
19 \\
11 \\
15\end{array}$ & $\begin{array}{r}5 \\
7 \\
17 \\
11 \\
20 \\
19\end{array}$ & $\begin{array}{l}0.66 \\
1.54 \\
0.20 \\
0.05 \\
0.03\end{array}$ & $\begin{array}{r}39.7 \\
60.6 \\
16.6 \\
5.3 \\
2.7\end{array}$ & $\begin{array}{r}16 \\
99 \\
73 \\
51 \\
61 \\
178\end{array}$ & \\
\hline 30 & $\begin{array}{l}0 \\
1 \\
4\end{array}$ & $\begin{array}{r}79 \\
18 \\
9\end{array}$ & $\begin{array}{l}19 \\
72 \\
69\end{array}$ & $\begin{array}{r}1 \\
6 \\
17\end{array}$ & $\begin{array}{l}1 \\
4 \\
5\end{array}$ & $\begin{array}{l}234 \\
643 \\
711\end{array}$ & $\begin{array}{l}71 \\
37 \\
59\end{array}$ & $\begin{array}{l}11 \\
14 \\
22\end{array}$ & $\begin{array}{l}13 \\
20 \\
24\end{array}$ & $\begin{array}{l}4.17 \\
0.01 \\
0.01\end{array}$ & $\begin{array}{l}80.7 \\
13.8 \\
11.6\end{array}$ & 29 & + \\
\hline 31 & $\begin{array}{l}0 \\
1 \\
4\end{array}$ & $\begin{array}{l}75 \\
74 \\
47\end{array}$ & $\begin{array}{l}22 \\
17 \\
45\end{array}$ & $\begin{array}{l}2 \\
6 \\
5\end{array}$ & $\begin{array}{l}1 \\
2 \\
3\end{array}$ & $\begin{array}{l}257 \\
146 \\
169\end{array}$ & $\begin{array}{r}104 \\
61 \\
110\end{array}$ & $\begin{array}{l}13 \\
22 \\
20\end{array}$ & $\begin{array}{r}9 \\
23 \\
12\end{array}$ & $\begin{array}{r}1.37 \\
1.82 \\
0.69\end{array}$ & $\begin{array}{l}57.8 \\
64.7 \\
40.9\end{array}$ & $\begin{array}{l}14 \\
26\end{array}$ & + \\
\hline $33^{*}$ & $\begin{array}{r}0 \\
2 \\
4 \\
6 \\
8 \\
10\end{array}$ & $\begin{array}{r}88 \\
55 \\
24 \\
7 \\
8\end{array}$ & $\begin{array}{r}10 \\
39 \\
72 \\
81 \\
42 \\
4\end{array}$ & $\begin{array}{r}1 \\
4 \\
2 \\
7 \\
35 \\
31\end{array}$ & $\begin{array}{r}1 \\
2 \\
2 \\
4 \\
15 \\
65\end{array}$ & $\begin{array}{l}322 \\
500 \\
965 \\
901 \\
482\end{array}$ & $\begin{array}{r}90 \\
96 \\
160 \\
96 \\
34 \\
93\end{array}$ & $\begin{array}{l}14 \\
20 \\
18 \\
16 \\
10 \\
17\end{array}$ & $\begin{array}{r}12 \\
6 \\
21 \\
15 \\
21 \\
17\end{array}$ & $\begin{array}{l}2.45 \\
0.27 \\
0.05 \\
0.08 \\
0.01\end{array}$ & $\begin{array}{r}71.0 \\
21.3 \\
4.9 \\
0.9 \\
0.1\end{array}$ & $\begin{array}{r}18 \\
37 \\
28 \\
39 \\
\\
252\end{array}$ & + \\
\hline 34 & $\begin{array}{l}0 \\
1\end{array}$ & $\begin{array}{l}73 \\
78\end{array}$ & $\begin{array}{l}23 \\
16\end{array}$ & $\begin{array}{l}3 \\
4\end{array}$ & $\begin{array}{l}1 \\
2\end{array}$ & $\begin{array}{l}429 \\
370\end{array}$ & $\begin{array}{r}150 \\
71\end{array}$ & $\begin{array}{l}24 \\
18\end{array}$ & $\begin{array}{r}24 \\
7\end{array}$ & $\begin{array}{l}1.11 \\
0.93\end{array}$ & $\begin{array}{l}52.6 \\
48.2\end{array}$ & 39 & + \\
\hline 35 & $\begin{array}{l}0 \\
2 \\
4\end{array}$ & $\begin{array}{l}85 \\
52 \\
18\end{array}$ & $\begin{array}{l}13 \\
26 \\
72\end{array}$ & $\begin{array}{r}1 \\
17 \\
6\end{array}$ & $\begin{array}{l}1 \\
4 \\
4\end{array}$ & $\begin{array}{l}422 \\
208 \\
600\end{array}$ & $\begin{array}{r}87 \\
104 \\
135\end{array}$ & $\begin{array}{l}25 \\
38 \\
25\end{array}$ & $\begin{array}{l}12 \\
12 \\
15\end{array}$ & $\begin{array}{l}1.25 \\
1.00 \\
0.05\end{array}$ & $\begin{array}{r}55.5 \\
50.0 \\
5.0\end{array}$ & $\begin{array}{l}25 \\
54\end{array}$ & + \\
\hline 36 & $\begin{array}{l}0 \\
2 \\
4 \\
6 \\
8 \\
9\end{array}$ & $\begin{array}{l}83 \\
83 \\
62 \\
26 \\
18 \\
17\end{array}$ & $\begin{array}{l}14 \\
12 \\
31 \\
68 \\
53 \\
35\end{array}$ & $\begin{array}{r}2 \\
3 \\
5 \\
4 \\
25 \\
35\end{array}$ & $\begin{array}{r}1 \\
2 \\
2 \\
2 \\
5 \\
13\end{array}$ & $\begin{array}{l}659 \\
386 \\
338 \\
246 \\
360 \\
482\end{array}$ & $\begin{array}{r}200 \\
102 \\
104 \\
96 \\
68 \\
44\end{array}$ & $\begin{array}{l}35 \\
20 \\
22 \\
14 \\
24 \\
14\end{array}$ & $\begin{array}{l}25 \\
18 \\
21 \\
13 \\
14 \\
22\end{array}$ & $\begin{array}{l}1.78 \\
1.83 \\
0.61 \\
0.15 \\
0.06 \\
0.04\end{array}$ & $\begin{array}{r}64.0 \\
64.8 \\
38.0 \\
12.9 \\
6.8 \\
4.8\end{array}$ & $\begin{array}{r}18 \\
39 \\
23 \\
59 \\
140 \\
192\end{array}$ & + \\
\hline 37 & $\begin{array}{l}2 \\
6\end{array}$ & $\begin{array}{l}70 \\
61\end{array}$ & $\begin{array}{l}25 \\
30\end{array}$ & $\begin{array}{l}3 \\
5\end{array}$ & $\begin{array}{l}2 \\
4\end{array}$ & $\begin{array}{l}317 \\
270\end{array}$ & $\begin{array}{l}113 \\
118\end{array}$ & $\begin{array}{l}22 \\
20\end{array}$ & $\begin{array}{l}21 \\
12\end{array}$ & $\begin{array}{l}1.94 \\
0.88\end{array}$ & $\begin{array}{l}66.6 \\
47.0\end{array}$ & $\begin{array}{l}24 \\
57\end{array}$ & + \\
\hline
\end{tabular}

* A distinction could not be made between components I and II on the final curves of dogs 29 , and 33 . 

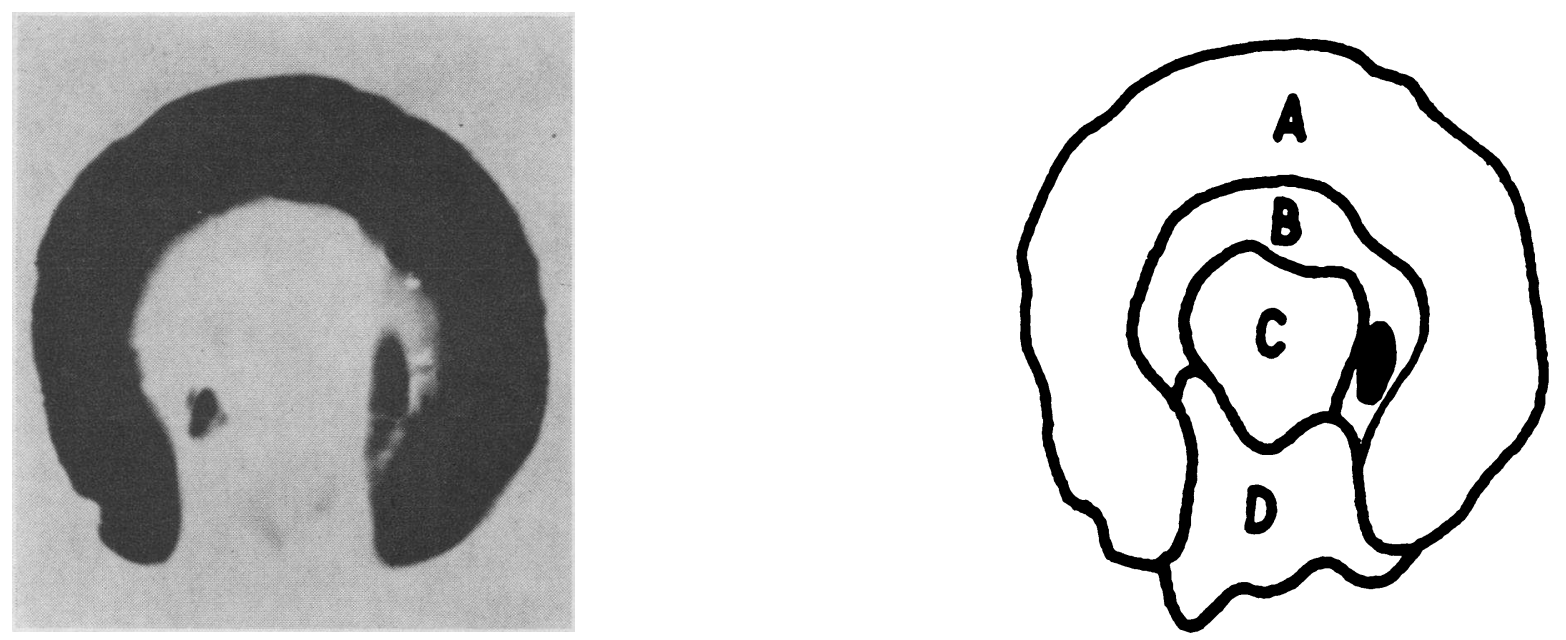

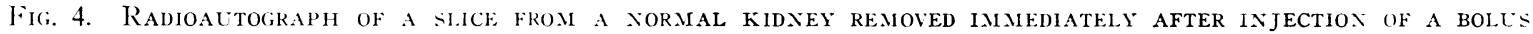
OF "KRYPTON INTO A RENAL ARTERT. Anatomical boundaries of the slices are shown alongside. $\mathrm{A}=\mathrm{cortex}, \mathrm{B}=$ outer medulla, $\mathrm{C}=$ inner medulla, $\mathrm{D}$ ) = renal fat. Black ovoid areas represent large blood vessels. Cortex was homogeneously perfused by component I, which had normal values on "washout" curves.

when compared to the normal kicheys within the first 7 days after laparotomy. Serial measurements made in 10 untreated homotransplanted dogs showed the same pattern of change in each case. There was a progressive diminution in the percentage of blood flow supplied to component I (Figure 2). This diminution occurred before there was a massive rise in blood urea nitrogen. The sequential relationships of the mean blood urea nitrogen and size of component $I$ in the group with homotransplanted kidneys are shown in Figure 3.

The progressive diminution in size of component I was associated with a relative decrease in tissue mass perfused by this component (Table VI). The blood flow rate in component I during and at the end of the rejection process differed from initial rates in individual dogs. In some dogs the blood flow rate more than doubled, whereas in others it declined to values less than control. In
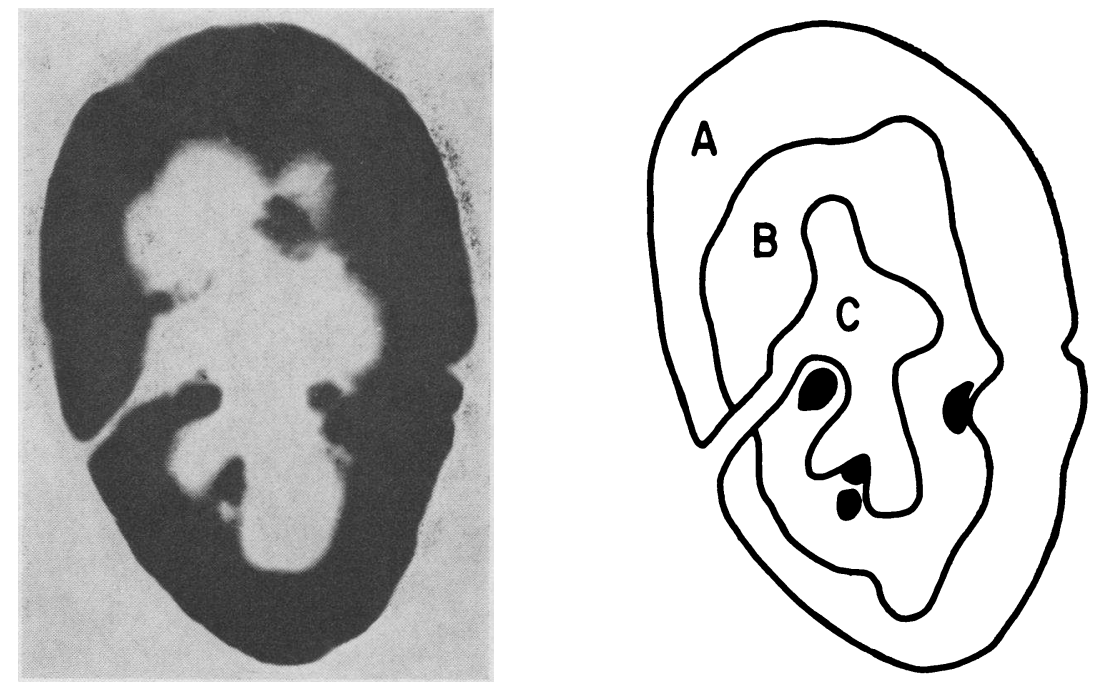

Fig. 5. Radioaltograph of a slice from an autotransplanted kidney. Experimental conditions and abbreviations are as in Figure 4. 
three kidneys component I was not apparent at the terminal uremic phase.

Radioautography. All kidneys were removed immediately after injection of ${ }^{85} \mathrm{krypton}$ and before significant washout of gas could occur. The greatest density of radioactivity in the renal parenchyma, therefore, occurred in the area of the highest flow (component I). A high density of radioactivity also appeared in the renal fat on some occasions, since its partition coefficient was nine times that of renal parenchyma. The fat also contained some residual activity from the ${ }^{133}$ xenon previously injected to record the associated washout curve.

The radioautographs of the four normal kidneys studied more than 7 days after the insertion of the renal arterial catheter are represented by Figure 4. The cortex was homogeneously filled with the greatest density of radiactivity. The same results were obtained with the group with three autotransplanted kidneys (Figure 5) and one homotransplanted kidney studied within 2 hours of operation.

When rejection commenced in dog 37 , analysis of the curve showed that component I had decreased from 70 to $61 \%$ of total renal blood flow and the percentage of kidney mass supplied by component I had decreased from 66.6 to $47.0 \%$.

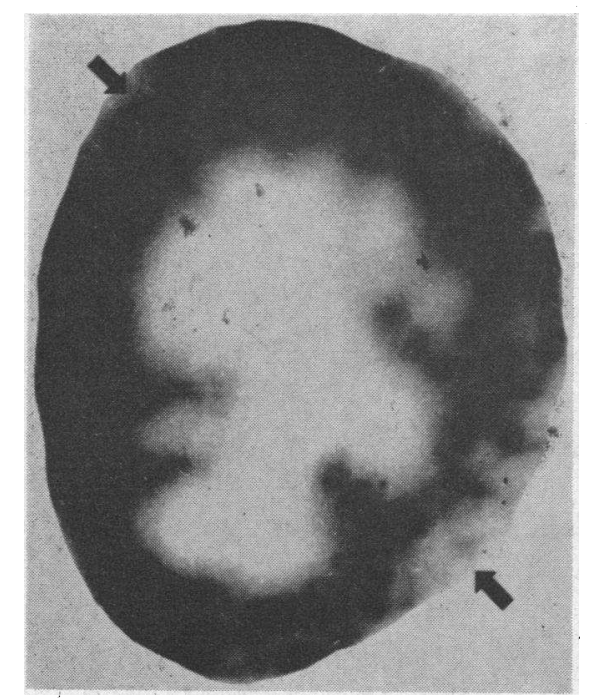

The radioautograph of the kidney at that time showed that the cortex contained component I, but some small areas of cortex also flowed at a slower rate (Figure 6). This finding confirmed the diminution in percentage of kidney mass supplied by component I. Figures $7 \mathrm{~A}$ and $\mathrm{B}$ show the radioautographs of two kidney slices from dog 35 . Component I was decreased to $18 \%$ of total renal blood flow and perfused $5.0 \%$ of kidney volume. The cortex still contained component I, but areas of cortex flowing at a slower rate had increased in prominence. Figures $8 \mathrm{~A}, \mathrm{~B}, \mathrm{C}$, and $\mathrm{D}$ show the radioautographs of four slices from the kidney of $\operatorname{dog} 36$. Component I had decreased to $17 \%$ of the total renal blood flow supplying only $4.8 \%$ of kidney mass. Radioautography did not locate the small mass of tissue supplied by this component. Exposure of the tissue slices was prolonged, and the fastest component to be demonstrated supplied the outer medulla. The cortex contained less concentration of radioactivity than the outer medulla and was therefore supplied by a slower blood flow. Figures $9 \mathrm{~A}, \mathrm{~B}$, and $\mathrm{C}$ are the radioautographs from three slices of the kidney of dog 33 . Components I and II were indistinguishable in the curve. The fastest flowing component on the radioautograph was predominantly in the outer medulla, with small areas of cortex supplied at the

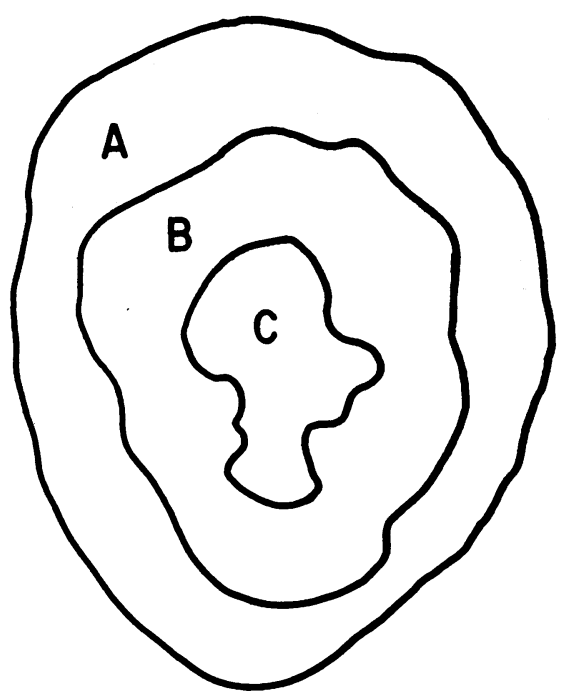

Fig. 6. RADIOAUTOGRAPH OF SLICE FROM THE UNTREATED REJECTING HOMOTRANSPLANTED KIDNEY OF DOG 37. Areas of cortex indicated by the arrows were not perfused by component I. The washout curve had shown that the percentage of total renal blood flow received by component I had decreased to $61 \%$ and the percentage mass of kidney perfused by component I had decreased to $47 \%$. 


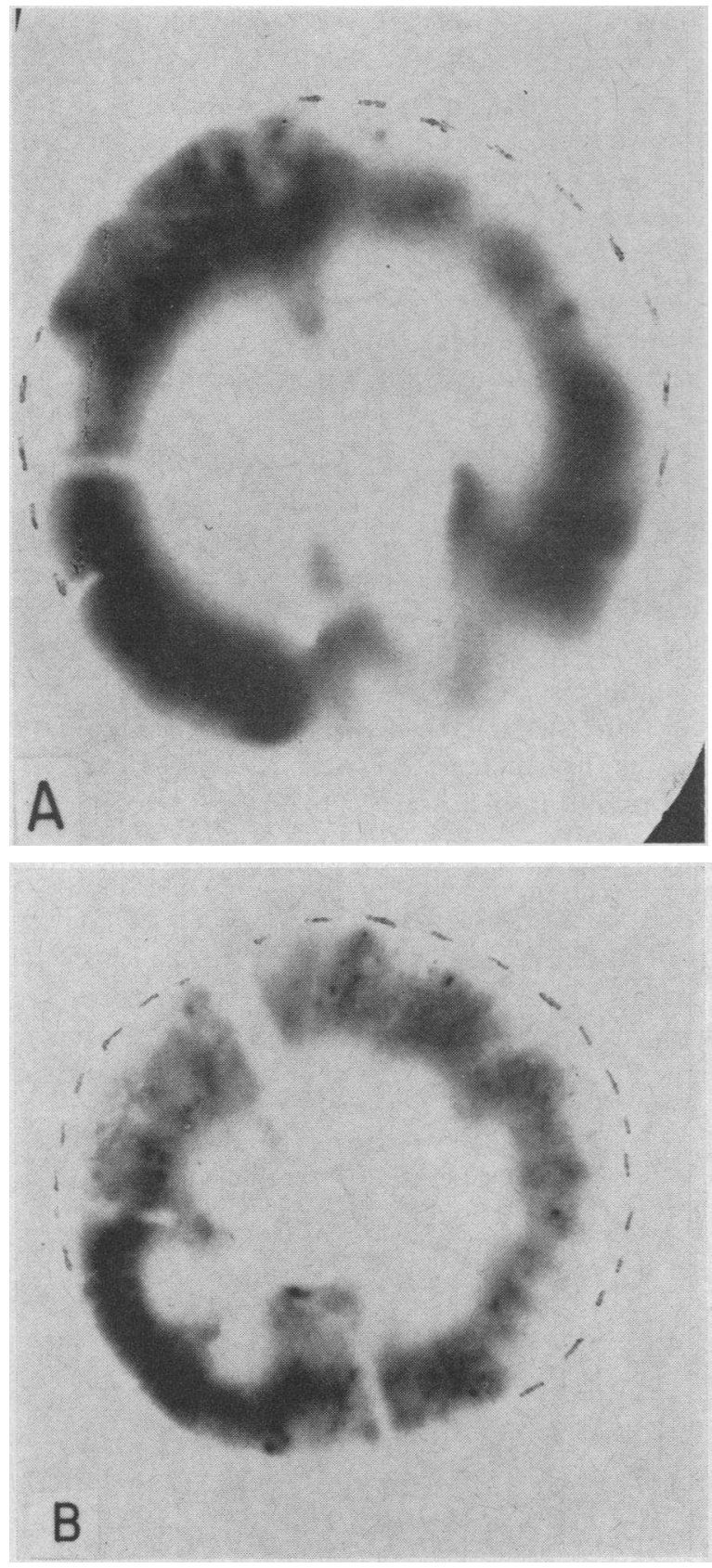

Fig. 7A \& B. Radionutographs From homotransPLANTED KIDNEY OF DOG 35. Large areas of cortex were not perfused by component I. External boundary of slice is defined by drawn interrupted line.

same rate. The concentration of radioactivity in the remainder of the cortex was less than that in the outer medulla. The majority of the cortex, therefore, received a slower flow than the outer medulla.

\section{Discussion}

The group of dogs with normal kidneys showed significantly different results for intrarenal distribution of blood flow when studied in the early and late periods after laparotomy for insertion of the catheter. After the arbitrary time intervals of 7 days after operation, the intrarenal distribution of blood flow remained stable. Observations made before the seventh postoperative day showed a decreased percentage of renal blood flow supplied to the fastest flowing component, but the flow rate per $100 \mathrm{~g}$ tissue supplied by this component was not significantly different. These data signified a decrease in the mass of tissue perfused by component $\mathrm{I}$. This conclusion is consistent with that of Carriere, Thorburn, O'Morchoe, and Barger (12). They demonstrated by radioautography that focal areas of cortex in the normal kidney may respond to trauma and hemorrhage by adopting the flow characteristics of component II.

These results demonstrated that the intrarenal circulations of the autotransplanted and normal kidneys behaved differently in response to certain stimuli when the measurements were made during anesthesia. Manipulation during laparotomy caused the normal kidney to redistribute its blood flow, whereas the distribution of blood flow in the autotransplanted kidney remained stable. This difference could be determined by denervation of the transplanted kidney. It has been shown that the kidney has afferent nerve fibers and the intrarenal vasculature is supplied by efferent sympathetic nerves (17). The preferential cortical ischemia may be associated with the richer innervation of the cortical vasculature (17). An alternative explanation could be that the period of ischemia during transplantation made the cortical vasculature unresponsive to manipulation. However, the evidence of Defalco and co-workers makes this explanation improbable (18).

The group of 12 dogs with first-set homotransplanted kidneys were subjected to ischemia for as long as 45 minutes. Within 2 hours of re-establishment of the renal circulation, the distribution of blood flow and blood flow per $100 \mathrm{~g}$ tissue in each component were not significantly different from those in the autotransplanted kidneys studied for as long as 77 days. Moreover, radioautographs of these kidneys did not demonstrate any 

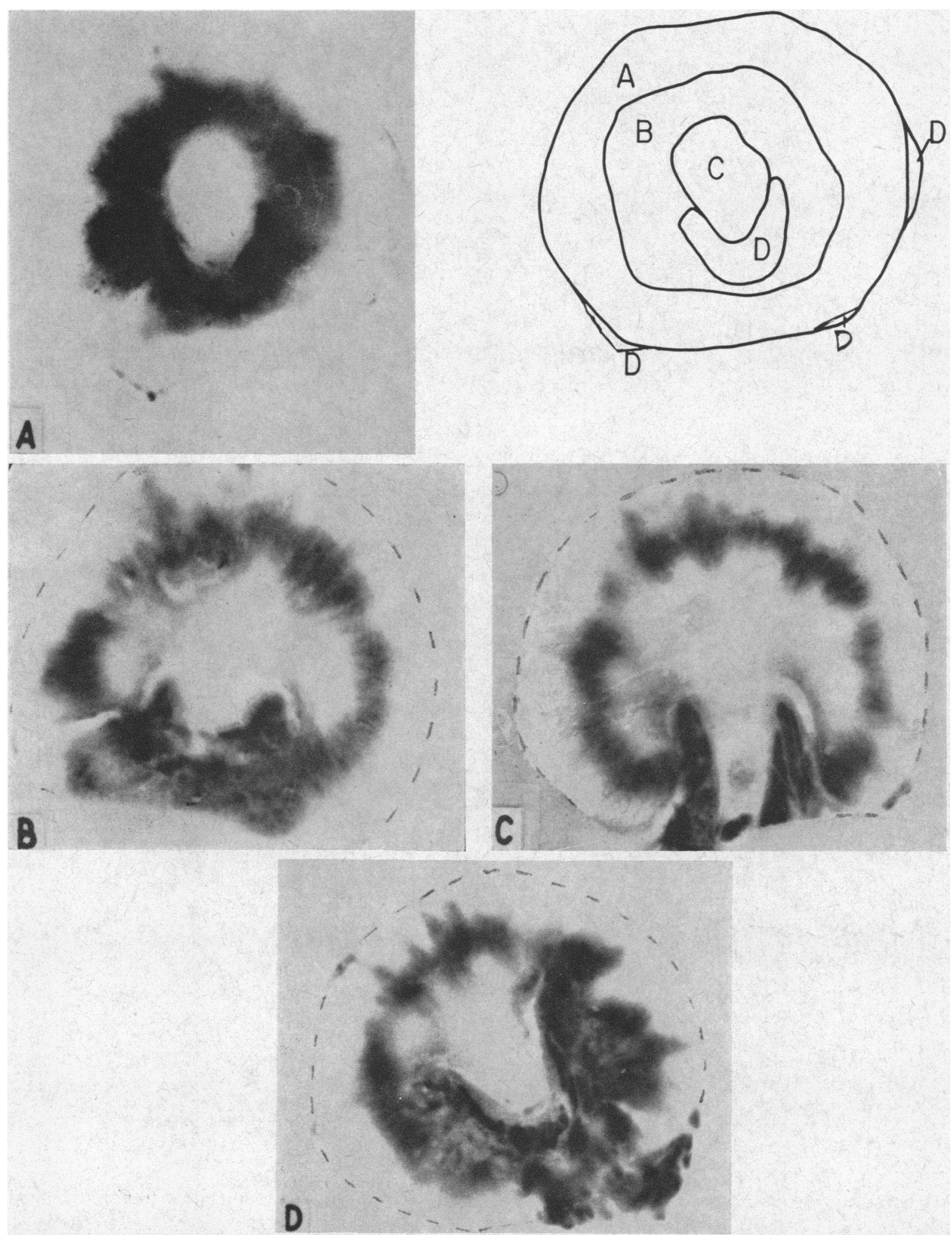

Fig. 8. A \pm RAdioautograph OF A SLICE OF THE HOMOTRANSPLANTED KIDNEY OF DOG 36 . External boundary of slice was drawn. Anatomical boundaries are shown alongside. $\mathrm{A}=$ cortex, $\mathrm{B}=$ outer medulla, $\mathrm{C}=$ inner medulla, $\mathrm{D}=$ fat. Fastest component of renal blood flow perfused outer medulla and adjacent areas of cortex.

$\mathrm{B}, \mathrm{C}, \& \mathrm{D}=$ RADIOAUTOGRAPHS OF OTHER SLICES OF THE SAME KIDNEY FROM DOG 36. 


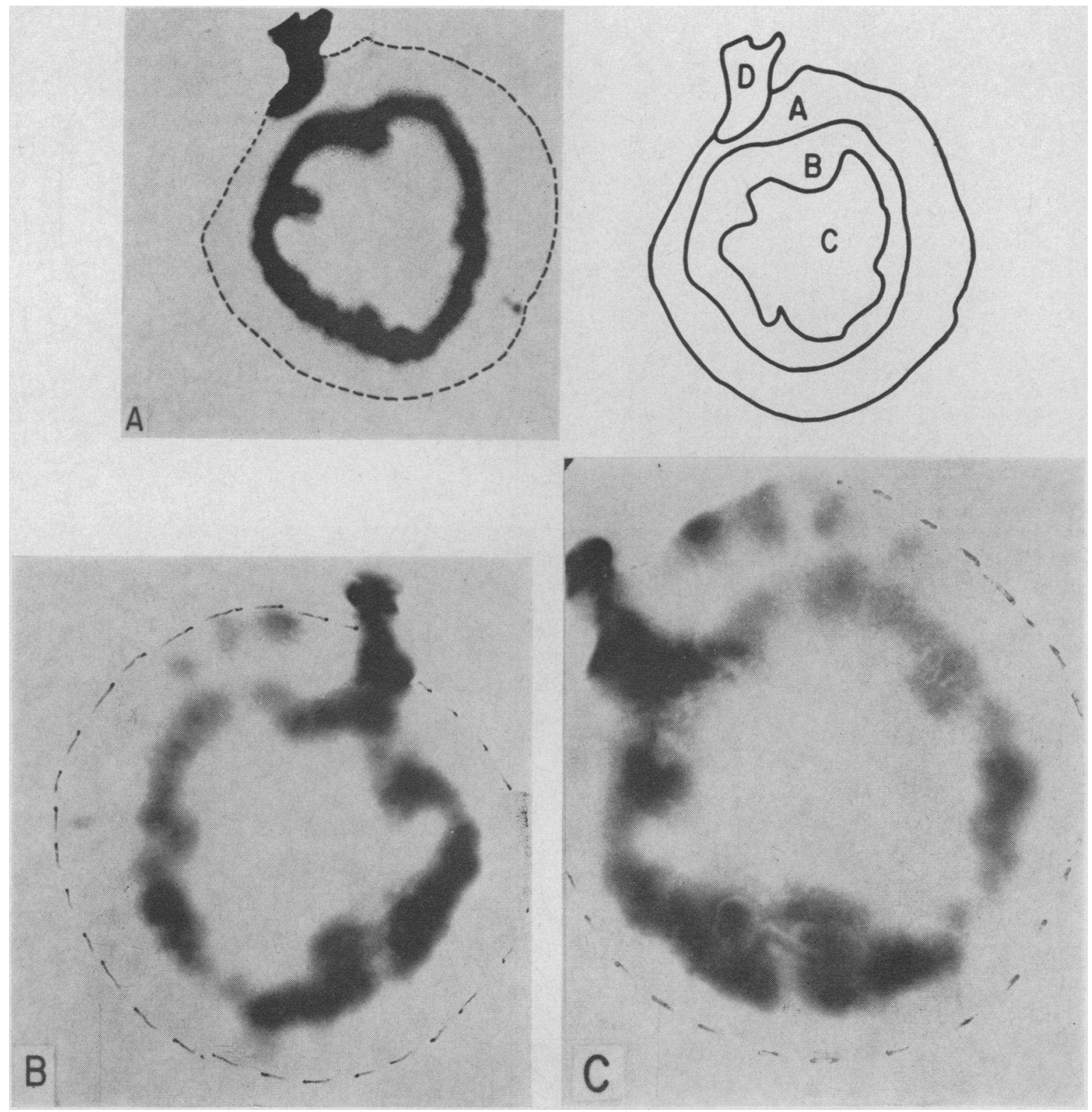

Fig. 9. A = RADioAutograph OF A KIDNEY SLICE FRoM the REJECTING KIDNEY OF DOG 33. External boundary was drawn. Anatomical boundaries of this slice are shown alongside. $\mathrm{A}=$ cortex, $\mathrm{B}=$ outer medulla, $\mathrm{C}=$ inner medulla, $\mathrm{D}=$ renal fat. Fastest flowing component of renal blood flow perfused outer medulla. Component I could not be distinguished from component II on the washout curve.

B \& $C=$ RADIOAUTOGRAPHS FROM OTHER SLICES OF THE KIDNEY OF DOG 33. Small areas of cortex supplied at approximately the same rate of blood flow as outer medulla were demonstrated.

heterogeneity of cortical perfusion, indicating that the total renal blood flow had assumed a stable value within 2 hours after homotransplantation. Other investigators $(3,5,19,20)$ have had re- sults that indicated a reduction of total renal blood flow within the first day after homotransplantation. However, they did not state the time interval that had elapsed between re-establishment 
of the circulation and their measurements, or the depth of anesthesia.

Cortical ischemia has previously been suggested to be associated with rejection of the homotransplanted kidney. Dempster $(1,19,21)$ injected pyelosil into the artery of the homotransplanted kidney after it had ceased to produce urine. He noted that very little of the dye entered the cortex, whereas in the autotransplanted kidney there was an even distribution of pyelosil throughout the cortex. Simonsen and associates (2) and Egdahl and Hume (22) reported that the cut surface of the anuric rejected homotransplanted kidney bled less than the normal kidney and suggested that anuria of rejection was accompanied by cortical ischemia.

Our studies have allowed serial measurements of intrarenal distribution of blood flow, and results have been obtained on a quantitative basis. The distribution of blood flow in the autotransplanted kidney when measured in dogs under pentobarbital anesthesia remained stable from the time of operation for periods as long as 77 days. The distribution in the homotransplanted kidney within 2 hours of operation was indistinguishable from that in the autotransplanted kidney. However, when rejection appeared, a progressively smaller percentage of total renal blood flow was supplied to the fastest flowing component. Radioautography demonstrated that this component occupied the cortex in the healthy normal and transplanted kidneys. When rejection occurred in the homotransplanted kidney, the fastest flowing component perfused a smaller volume of cortical tissue and eventually moved to the outer medulla. The latter observation demonstrated that in abnormal circumstances the fastest flowing component is not necessarily limited to the cortex.

The importance of measuring both the blood flow rate to a component $(\mathrm{k})$ and the percentage of total renal blood flow supplied to that component $\left(A_{0}\right)$ has been demonstrated, since these two parameters have been shown to vary independently of each other. The blood flow rate in component I fluctuated markedly as rejection progressed, but the percentage of total renal blood flow supplied to this component consistently decreased. These results were explained by postulating that heterogeneity of blood flow rate occurred in the cortex during rejection. This explanation was confirmed by radioautography. The correlation between the predicted mass of tissue supplied by component I and the mass determined by radioautography is further evidence of the validity of measuring the intrarenal distribution of blood flow by the inert gas washout technique.

The mechanism of the vascular changes during rejection is open to speculation. One concept maintains that these changes result from compression due to interstitial edema and cellular infiltration. Under these circumstances, the pressure might be evenly distributed throughout the kidney and the blood would take the shortest pathway through the kidney. It would then pass through the areas of cortex closest to the arcuate arteries, leaving the more superficial cortex ischemic. The ischemia would consequently progress concentrically from the surface of the cortex. However, our radioautographic evidence does not show a concentric advance of the ischemia and, therefore, does not support this concept.

Another mechanism may involve the damage of vessels by immunologically stimulated cells. Kountz and coworkers (3) have suggested blockage and damage of cortical vessels by collection of lymphocytes and pyroninophyllic cells in the peritubular capillaries and venules. Porter and co-workers (23) confirmed their findings and postulated that these products of cellular reaction would occur predominantly where the circulation is most sluggish. However, there is no evidence that the circulation of the cortical peritubular capillaries is more sluggish than that in the peritubular capillaries of the outer medulla.

A third concept maintains that the vascular reaction may be humoral in origin, due to circulating antibodies or diffusion of substances produced locally in the kidney. The general subject of circulating antibodies found in homograft recipients has been well reviewed (24-29). The importance of the vascular system as an end organ for immunological damage has been presented by Horowitz and his associates (30). They produced evidence which suggested that antigen-antibody complexes appear in the arteries and arterioles of the transplant during rejection.

The early diagnosis of rejection of a homotransplanted kidney remains a problem. Pyrexia associated with rejection may be confused with local or systemic infection. Oliguria may be due to leakage or obstruction in the urinary outflow 
tract. An increase in immunosuppressive therapy would be detrimental if infection or urine leakage were present. Redistribution of renal blood flow occurred early during the course of rejection and could be used as an early diagnostic test. However, it is not pathognomonic for rejection since it has been noted under other circumstances. It occurred in three autotransplanted kidneys not reported in this series. Two of these kidneys had renal arterial thrombosis and one had renal venous thrombosis. Redistribution has also been shown to occur in normal and transplanted kidneys during severe hemorrhagic hypotension $(12,31,32)$, but these circumstances can be readily distinguished from rejection with other criteria.

\section{Acknowledgments}

We wish to express our thanks to A. Clifford Barger and J. Alan Herd and their associates for their interest and valuable discussion.

\section{References}

1. Dempster, W. J. Kidney homotransplantation. Brit. J. Surg. 1953, 40, 447.

2. Simonsen, M., J. Buemann, A. Gammeltoft, F. Jensen, and $\mathrm{K}$. Jøergensen. Biological incompatibility in kidney transplantation in dogs. I. Experimental and morphological investigations. Acta path. microbiol. scand. 1953, 32, 1.

3. Kountz, S. L., M. A. Williams, P. L. Williams, G. Kapros, and W. J. Dempster. Mechanism of rejection of homotransplanted kidneys. Nature (Lond.) 1963, 199, 257.

4. Jackson, B. T., and J. A. Mannick. Serial blood flow in first set renal homotransplants undergoing rejection. Surg. Gynec. Obstet. 1964, 119, 1265.

5. Jones, H. B. Respiratory System: Nitrogen Elimination in Medical Physics, vol. 2, O. Glasser, Ed. Chicago, Year Book, 1950, pp. 855-871.

6. Thorburn, G. D., H. H. Kopald, J. A. Herd, M. Hollenberg, C. C. C. O’Morchoe, and A. C. Barger. Intrarenal distribution of nutrient blood flow determined with krypton ${ }^{85}$ in the unanesthetized dog. Circulat. Res. 1963, 13, 290.

7. Dobson, E. L., and G. F. Warner. Measurement of regional sodium turnover rates and their application to the estimation of regional blood flow. Amer. J. Physiol. 1957, 189, 269.

8. Defares, J. G., and I. N. Sneddon. An Introduction to the Mathematics of Medicine and Biology. Chicago, Year Book, 1960, pp. 582-590.

9. Kety, S. S. The theory and applications of the exchange of inert gas at the lungs and tissues. Pharmacol. Rev. 1951, 3, 1.
10. Conn, H. L., Jr. Equilibruim distribution of radioxenon in tissue; xenon-haemoglobin association curve. J. appl. Physiol. 1961, 16, 1065.

11. Ladefoged, J., F. Pederson, U. Doutheil, P. Deetjen, and E. E. Selkurt. Renal blood flow measured with xenon ${ }^{133}$ wash-out technique and with an electromagnetic flowmeter. Pflügers Arch. ges. Physiol. $1965,284,195$.

12. Carriere, S., G. D. Thorburn, C. C. C. O'Morchoe, and A. C. Barger. Intrarenal distribution of blood flow in dogs during hemorrhagic hypotension. Circulat. Res. 1966, 19, 167.

13. Rosen, S. M., N. K. Hollenberg, and A. B. Retick. Measurement of compartmental distribution of blood flow and total blood flow in homotransplanted dog kidney using $\mathrm{Xe}^{133}$. washout techniques and electromagnetic flowmeter. In preparation.

14. Herd, J. A., and A. C. Barger. Simplified technique for chronic catheterization of blood vessels. J. appl. Physiol. 1964, 19, 791.

15. Chidsey, C. A. III, H. W. Fritts, Jr., A. Hardewig, D. W. Richards, and A. Cournand. Fate of radioactive krypton $\left(\mathrm{Kr}^{85}\right)$ introduced intravenously in man. J. appl. Physiol. 1959, 14, 63.

16. Ueda, K., P. R. Lichtlen, J. R. Rees, R. S. Ross, and M. Iio. Coronary blood flow measurement in dog and man using $\mathrm{Kr}^{85}$ or $\mathrm{Xe}^{133}$ (abstract). Fed. Proc. 1963, 22, 641 .

17. Mitchell, G. A. G. The intrinsic renal nerves. Acta anat. (Basel) 1951, 13, 1.

18. Defalco, A. J., E. D. Mundth, L. Brettschneider, Y. G. Jacobson, and J. E. McClenathan. A possible explanation for transplantation anuria. Surg. Gynec. Obstet. 1965, 120, 748.

19. Dempster, W. J. The anurias following kidney transplantation. Acta med. scand. 1954, 148, 91.

20. Mims, M. M. Hemodynamics of renal transplantation. J. Urol. (Baltimore) 1961, 86, 493.

21. Dempster, W. J. A consideration of the cause of functional arrest of homotransplanted kidneys. Brit. J. Urol. 1955, 27, 66.

22. Egdahl, R. H., and D. M. Hume. Immunologic studies in renal homotransplantation. Surg. Gynec. Obstet. 1956, 102, 450.

23. Porter, K. A., N. H. Joseph, J. M. Rendall, C. Stolinski, R. J. Hoehn, and R. Y. Calne. The role of lymphocytes in the rejection of canine renal homotransplants. Lab. Invest. 1964, 13, 1080.

24. Merrill, J. P., C. Hanau, and M. D. Hawes. A demonstration of a cytotoxic effect in vitro following the rejection of skin grafts by the rabbit. Ann. N. Y. Acad. Sci. 1960, 87, 266.

25. Medawar, P. B. Reactions to homologous tissue antigens in relation to hypersensitivity in Cellular and Humoral Aspects of the Hypersensitive States, H. Sherwood Lawrence, Ed. New York, Hoeber, 1959, pp. 504-529.

26. Stetson, C. A., and E. Jensen. Humoral aspects of the immune response to homografts. Ann. N. Y. Acad. Sci. 1960, 87, 249. 
27. Medawar, P. B. The immunology of transplantation. Harvey Lect. 1956-57, 52, 144.

28. Lawrence, H. S. Homograft sensitivity An expression of the immunologic origins and consequences of individuality. Physiol. Rev. 1959, 39, 811.

29. May, J., L. W. Way, and J. S. Najarian. Onset, duration, and mechanism of antigen release from the homotransplanted kidney. Surg. Forum 1965, $16,250$.

30. Horowitz, R. E., L. Burrows, F. Paronetto, D. Dreiling, and A. E. Kark. Immunologic obser- vations on homografts. II. The canine kidney. Transplantation 1965, 3, 318.

31. Rosen, S. M., B. Truniger, H. R. Kriek, D. E. Oken, J. E. Murray, and J. P. Merrill. Intrarenal distribution of blood flow in normal and autotransplanted dog kidneys. Effect of hemorrhagic hypotension and mannitol (abstract). J. clin. Invest. 1965, 44, 1092.

32. Rosen, S. M., B. Truniger, H. R. Kriek, D. E. Oken, J. E. Murray, and J. P. Merrill. Regional distribution of blood flow in the denervated kidney during hemorrhagic hypotension (abstract). Clin. Res. 1965, 13, 558. 